\title{
Changes 1986-1990 in the list of Finnish insects
}

\author{
Hans Silfverberg
}

Silfverberg, H.: Changes 1986-1990 in the list of Finnish insects. - Entomol. Fennica 2:9-17. 1991.

During the period 96 insect species have been deleted from the list of Finnish insects, and 419 species have been added. By the end of 1990, a total of 19213 insect species were known from Finland.

Hans Silfverberg, Zoological Museum, N. Järnvägsgatan 13, 00100 Helsingfors, Finland.

This paper is a sequel to a similar list published in 1986 (Silfverberg, Notulae Entomol. 66:131-152), which itself was a continuation of a series of lists published by Hellén and Silfverberg in 19211981. Insect species new to the fauna of Finland during the most recent five-year period are listed here, with references, as are those deleted from the list. A few previously unnoted changes have also been included.

For the new species the locality has generally been given, with its biogeographical province. If the species has been reported from many localities, often only the provinces have been listed, or the abbreviation m.l. (= many localities) has been used. When Finland is mentioned as the locality, the original record gave a no more exact location.

Some other abbreviations or signs must also be explained. Thus f.d. $=$ false determination and sp.dist. $=$ distinct species ( previously considered a variety, a synonym or such like); in literature references NE = Notulae Entomologicae. A species enclosed in square brackets is considered accidentally imported, and has not been included in the species total. Again square brackets have been employed for the name used in the original record when different from the one used here. Other symbols are $*=$ new to science and ! = differing from the check-list mentioned for the order.

During the period covered by this list there have been 96 species deleted from the Finnish fauna, and 419 species added to it. At the end of 1990 a total of 19213 insect species were known from Finland.

\section{Collembola}

Species reported and later deleted

Arrhopalites cochlearifer Gisin (f.d., = A. spinosus Rusek) N: Tuusula, Ta: Tammela. Huhta \& al. 1986 Ann. Zool. Fennici 23:359. Vilkamaa 1989 NE 69:64.

\section{Additions}

Anurida brunsvigiensis Hüth. Oa: Kuortane (Laitakari). Vilkamaa 1989 NE 69:63.

Onychiurus fimatus Gisin Ta: Lammi (Uusitalo). ibid.:63. Tullbergia arctica Wahlgr. Li: Utsjoki (Koponen). ibid.:63. Mesaphorura hylophila Rusek Ta: Lammi. ibid.:63.

— tenuisensillata Rusek Li: Utsjoki (Koponen). ibid.:63. Anurophorus laricis Nicol. Ab: Pargas (Niemi), N: Tvärminne (Vuorimies). ibid.:63.

Folsomia lawrencei Rusek Ta: Lammi (Uusitalo). ibid.:64. — sensibilis Ksenem. Li: Utsjoki (Koponen). ibid.:64.

- spinosa Ksenem. Ta: Lammi. ibid.:64.

Proisotoma abiskoensis Agrell Li: Utsjoki. Koponen 1984 Oulanka Rep. 4:59.

Vertagopus pseudocinereus Fjellb. Li: Utsjoki (Koponen). Vilkamaa 1989:64.

Isotomatshernovi Martyn. Li: Utsjoki(Hurme \& Koponen). ibid.:64.

Arrhopalites spinosus Rusek (cochlearifer auct.) N: Tuusula, Ta: Tammela. ibid.:64.

— pseudoappendices Rusek N: Lohja (Biström \& Hippa). ibid.:64.

Present number: $192+14=206$ spp. 


\section{Ephemeroptera}

Additions

Baetis liebenauae Keff. Ok: Kuhmo (Lyytikäinen), Savolainen \& Pulkkinen 1987 Kulumus 9:17.

Present number: $49+1=50$ spp.

\section{Psocoptera}

Deletions

Liposcelis kidderi (Hag.) (f.d., = L. pearmani Lienh.). Lienhard 1990 Zool. Jahrb., Syst. 117:157.

Additions

*Liposcelis pearmani Lienh. (L. kidderi auct. nec (Hag.)) Finland. Lienhard 1990 Zool. Jahrb., Syst. 117:157.

Trichadenotecnumincognitum Roesl.Tb: Saarijärvi(Biström \& Väisänen). Meinander 1986 NE 66:184.

Present number: $60-1+2=61$ spp.

\section{Heteroptera}

Deletions

Orthocephalus coriaceus $(\mathrm{F}).(\mathrm{f} . \mathrm{d} .,=$ O. vittipennis $(\mathrm{H} .-\mathrm{S})$.$) .$ Lammes \& Rinne 1990 Entomol. Fennica 1:210.

Monosynamma nigritula (Zett.) (f.d., = M. bohemani (Fall.). ibid.:210.

Elatophilus nigrellus (Zett.) (= E. nigricornis (Zett.)). ibid.:210.

Additions

*Polymerus tepastus Rinne Al, Ab, N, Ka, St, Ta, Sa, Kl, Oa, $T b, K b, O b, K s, L k, L i$. Rinne 1989 Ann. Entomol. Fennici 55:98.

* lammesi Rinne $A b$ : Korpo Jurmo, Dragsfjärd Örö. ibid. 99.

*Trigonotylus fuscitarsis Lammes $\mathrm{Sa}, \mathrm{Tb}, \mathrm{Sb}, \mathrm{Ok}, \mathrm{Ob}, \mathrm{Ks}$, Lk, Le, Li. Lammes 1987 Ann. Entomol. Fennici 53:69.

[Castolustricolor Champ. $N$ : Helsinki(I.Kullberg). Koponen 1988 NE 68:150.]

Present number: $478-3+3=478$ spp.

\section{Homoptera}

Deletions

Chaitophorus truncatus (Hausm.) (f.d., = C. nigricantis Pint.). Pintera 1987 Deutsche Entomol.Zeitschr. 34:330.

Pterocomma populeum (Kalt.) Doubtful record. Heie 1986 Fauna Entomol. Scand. 17:23.

- ringdahli Wahlgr. (= P. rufipes (Hart.)). ibid.:24.

Additions

Aphrodes bicincta (Schr.) ObN: Pello, LkE: Savukoski. Raatikainen \& Ylönen 1988 Ann. Entomol. Fennici $54: 74,85$.
Edwardsiana tersa (Edw.) ObN, LkE, Li. ibid.:74, 85. Psammotettix excisus (Mats.) LkW, LkE. ibid.:74, 87. Bemisia tabaci (Genn.) Finland. Tiittanen 1988 Kasvinsuojelulehti 21:15.

*Chaitophorusfurcatus Pint. Tb: Rautalampi (Heikinheimo). Pintera 1987 Deutsche Entomol. Zeitschr. 34:239.

* nigricantis Pint. $N$ : Tikkurila (Heikinheimo), Tb: Rautalampi (Heikinheimo). ibid.:279.

*Schizaphis thunebergi Heie Ka: Vehkalahti (Thuneberg). Heie 1986 Fauna Entomol. Scand. 17:72.

Aphis podagrariae Schrank Ta. ibid.:217.

- pseudocomosa Stroyan $A l, A b$. ibid.:226.

Dysaphis aizenbergi (Shap.) N: Vantaa. Heikinheimo 1990 Entomol. Fennica 1:65.

- hirsutissima (Börn.) N: Vantaa. ibid.:67.

- calamagrostis (Oss.) N, Ka, Sa, Ob. ibid.:68.

Hyadaphis passerinii (Guerc.) Ab: Perniö, N: Helsinki \& Vantaa. ibid.:69.

- polonica Szeleg. N: Helsinki. ibid.:69.

- mosana HRL. Ab: Nauvo. ibid.:70.

Pseudacaudella rubida Börn. Ob: Rovaniemi. ibid.:73. Fimbriaphis latifrons (Börn.) Ks, Lk, Li. ibid.:73.

Myzodium modestum (Hott.) Ob: Rovaniemi. ibid.:76. Capitophorus carduinus (Walk.) Al, N, Ka. ibid.:76.

Nasonovia vannesi Stens. Ks: Kuusamo (P.Alanko). ibid.:78. Hyperomyzus zirnitsi boerneri Prev.Ob: Rovaniemi.ibid.:79.

Rhopalosiphoninus calthae (Koch) $\mathrm{Ta}$ : Janakkala, Sa: Mikkeli. ibid.:80.

Aulacorthum flavum (Müll.) N, St, Sa, Tb, Sb, Om, Ob, Ks, $L k$. ibid.:81

— speyeri (Börn.) Sa: Kouvola (J.Halme). ibid.:82.

- vaccinii HRL. Ks, Lk, Li. ibid.:83.

Acyrtosiphon brachysiphon HRL. Tb: Rautalampi \& Vimpeli, $O k$ : Kuhmo. ibid.:84.

- knechteli (Börn.) Al,St,Ta,Tb,Sb,Om,Ks,Lk. ibid.:85.

— chelidonii (Kalt.) N: Helsinki (L.Huldén), ibid.:87.

Metapolophium tenerum HRL. N: Vantaa, Ob: Rovaniemi. ibid.:87.

Cryptaphis poae (Hardy) N: Vantaa. ibid..88.

Subacyrtosiphon cryptobium HRL. N: Vantaa. ibid.:90.

Rhodobium porosum (Sanders.) Sa: Mikkeli (V.Kanervo). ibid.:90.

Sitobion dryopteridis (Holm.) Ta: Riihimäki (E.A.Oinonen). ibid.:92.

- graminearum (Mordv.) N: Vantaa. ibid.:93.

Uroleucon riparium (Stroyan) St: Yläne, Ks: Kuusamo. ibid.:95.

Present number $761-3+35=793$ spp.

\section{Thysanoptera}

Additions

Frankliniella occidentalis (Perg.) m.l. (Ab-Ob). Tiittanen 1988 Kasvinsuojelulehti 21:15. Brax \& Tiittanen 1989 Kasvinsuojelulehti 22:21. Tiittanen \& Markkula 1989 Acta Entomol. Fennica 53:59.

Present number $113+1=114$ spp. Coleoptera 


\section{Deletions}

Bradycellus harpalinus (Serv.) Doubtful record. Lindroth \& al. 1986 Fauna Entomol. Scand. 15:380.

Ochthebius auriculatus Rey Doubtful record. Hansen 1987 Fauna Entomol. Scand. 18:39.

Anacaena limbata (F.) (f.d., = A. lutescens (Steph.)). ibid.: 173 .

Laccobius striatulus (F.) (f.d., = L. simuatus Motsch.) Clayhills 1986 NE 66:185.

Carpelimusheidenreichi (Ben.) (f.d., =C. lindrothi $($ Palm)). Rutanen \& Rassi 1990 NE 69:212.

Leptusa saalasi Kang. (=L. pulchella (Mannh.)). Lundberg 1986 Entomol. Tidskr. 107:149. Pace 1989 Mem. Mus. Civ. Stor. Nat. Verona (II. ser.) (A) 8:85

Rybaxis laminata (Motsch.) $(=R$. longicornis (Leach)). Besuchet 1989 Die Käfer Mitteleuropas 12:243.

Atomaria attila Rtt. (f.d.). Kangas 1987 NE 67:212.

- peltatula Rtt. (f.d,; recorded outside Finland). ibid.:212.

Ropalodontus perforatus (Gyll.) (f.d., = R. strandi Lohse). Rutanen 1987 NE 67:213.

Mordellistena dalmatica Erm. (f.d.,=M.praesagita Kangas). Kangas 1988 Ann. Entomol. Fennici 54:89.

Phyllotreta cruciferae (Gze.) Doubtful record. Silfverberg 1990 NE 69:213.

Dorytomus nordenskioldi Faust Recorded outside Finland. Silfverberg 1987 NE 67:213.

Tychius crassirostris Kirsch Doubtful record. Mannerkoski 1986 NE 66: 181

Additions

Bembidion aeneum Germ. Ks: Kuusamo. Muona 1990 NE 69:195.

Ochthebius kaninensis Popp. (sp.dist.) Ks: Kuusamo. Hansen 1987 Fauna Entomol. Scand. 18:42.

*Hydrochus megaphallus Berge Hen. Kb: Liperi (Palmén). Berge Henegouwen 1988 Balfour-Browne Club Newsl. 42:19.

Helophorus griseus Hbst. Al. Hansen 1987:116.

Anacaena lutescens (Steph.) (A. limbata auct. nec F.) (sp.dist.). ibid.: 173

- limbata (F.) (nec lutescens) Ab: Karjalohja, Karis \& Lojo. Rutanen 1990 NE 69:212.

Laccobius sinuatus Motsch. (L. striatulus auct. nec F.) Al,N, Ta. Clayhills 1986 NE 66:185.

Enochrus fuscipennis (Thoms.) (sp.dist.) m.l. Hansen 1987:200.

Ptilium affine Er. Ab: Perniö. Y.Kangas 1987 NE 67:214.

Quedius puncticollis Thoms. N: Helsinki, Ta: Loppi. Rutanen 1987 NE 67:210.

Gyrohypnus liebei Scheerp. (ater auct. nec Steph.) (sp.dist.). Mahler 1987 Entomol. Meddel. 54:197; Lohse 1989 Käfer Mitteleur. 12:156.

Ochthephilum collare (Rtt.) N: Espoo (Dahlgren). Muona 1988 NE 68:25.

Stenus longitarsis Thoms. Ab: Pargas. Clayhills 1987 NE $67: 210$.

Thinobius brevipennis Kiesw. Ks: Kuusamo. ibid.:25.
Bledius fontinalis Bernh. Ks: Kuusamo, Li: Utsjoki. Herman 1986 Bull. Amer. Mus. Nat. Hist. 184:193. Clayhills 1988 NE 68:150.

- pallipes (Grav.) Li: Ivalo. Herman 1986:195.

Mycetoporus erichsonanus Fag. Li: Utsjoki. Clayhills 1988 NE 68:150.

Lordithon trinotatus (Er.) N: Siuntio \& Helsinki. Muona 1990 NE 69:195.

Tachyporus dispar (Payk.) (sp.dist.) Al-Ob. Silfverberg 1990 NE 69:210.

Oxypoda lapponica Lundb. Kb: Rääkkylä. Clayhills 1988 NE 68:151.

Ischnoglossa elegantula (Mannh.) (sp. dist.) m.l. Wunderle 1990 Entomol. B1. 86:57.

Dinarda maerkelii Kiesw. (sp.dist.).Zerche 1989 Die Käfer Mitteleuropas 12:222.

- hagensii Wasm. (sp.dist.). ibid.:222.

Lundbergia trybomi (J.Sahlb.) Li: Inari (Trottestam \& Muona). Muona 1988 NE 68:25.

Atheta insecuta Epp. Ks: Kuusamo. Muona 1990 NE 69:195.

*Amischa andreasi Muona Ks: Kuusamo, Oulanka. Muona 1990 Entomol. Tidskr. 111:20.

- bifoveolata (Mannh.) Al, Ab, N, Ka, St, Ta, Sa, Tb, Kb, $O b, K s, L k, L i$. ibid.:24.

Aphodius paykulli Bed. Al: Föglö. Hanski \& Kuusela 1983 Acta Entomol. Fennica 42:36.

Melanophila formaneki (Jacobs.) St: Harjavalta. Heliövaara, Väisänen \& Mannerkoski 1990Entomol. Fennica 1:221.

Atomaria ihsseni Johns. Kb: Ilomantsi. Kangas 1987 NE $67: 212$.

- grossepunctata Rtt. Ks: Kuusamo. ibid.:212.

Scymmus femoralis Gyll. Ab, N, St, Ta. Rutanen 1988 NE 68:152.

*Nephus rutaneni Fürsch Ta: Hausjärvi (Rutanen). Fürsch 1986 Ann. Entomol. Fennici 52:107.

- limonii (Donisth.) Ab:Kustavi. Clayhills $1986 \mathrm{NE}$ 66: 186.

Hyperaspis inexpectata Günth. Ab: Turku, Ta: Riihimäki. Clayhills \& Rutanen 1987 NE 67:209.

Melanophthalma suturalis (Mannh.) (sp.dist.). N: Hanko. Y.Kangas 1987 NE 67:214.

Orthocis vestitus (Mell.) Ab, Ta. Muona 1988 NE 68:26.

Ropalodontus strandi Lohse (perforatus auct.). Rutanen 1987 NE 67:213.

[Cylindronotus dermestoides (I1l.) $N$ : Helsingfors (M.S.Meriluoto). Biström 1988 NE 68:130.]

Cyrtanaspis phalerata (Germ.) N: Mäntsälä. Rutanen 1990 NE 69:213.

Mordella brachyura Muls. N: Hyvinkää. ibid.:213.

* Mordellistena multicicatrix Kangas Al: Eckerö. Kangas 1986 Ann. Entomol. Fennici 52:139.

* praesagita Kangas (M.dalmatica auct. nec Erm.) Ab, Ka, Sa. Kangas 1988 Ann. Entomol. Fennici 54:89.

[Tetropium gabrieli Wse. N: Helsinki.Jansson 1990 Entomol. Fennica 1:118.]

Galerucella sagittariae (Gyll.) (sp.dist.). Hippa \& Koponen 1986 Ann. Entomol. Fennici 52:49.

Longitarsus kutscherai (Rye) N, Ka, Tb. Rutanen 1987 NE $67: 213$. 
Altica lythri Aubé Ab: Pargas, Ka: Virolahti. Clayhills 1988 NE 68:152.

Sphaeroderma testaceum (F.) Al: Lemland. Clayhills 1986 NE 66:187.

Liophloeus lentus herbsti Gyll. Al: Maarianhamina. Kangas 1990 NE 69:214.

Hypera fornicata (Pen.) Ka: Imatra. Clayhills 1986 NE 66:187.

Dorytomus ictor (Hbst.) N: Espoo. Mannerkoski 1987 NE 67:213.

Tychius breviusculus Desbr. N: Helsinki. Mannerkoski 1986 NE 66:180.

Rhynchaenus pseudostigma Temp. $N$ : Helsinki, Ta: Luopioinen. Kangas 1990 NE 69:213

Ceutorhynchus thomsoni Kolbe $N$ : Siuntio. Muona 1988 NE 68:26.

- granulicollis Thoms. N: Pyhtää. ibid.:26.

— melanostictus (Marsh.) Kb: Pyhäselkä. Clayhills 1990 NE 69:214.

— pallidicornis Goug. \& Bris. N: Pukkila. Rutanen 1988 NE 68:153.

Trypodendron proximum (Nij.) (piceum Strand) Ks: Kuusamo. Muona 1990 NE 69:197.

Included by mistake in 1979 checklist

Carpelimus subtilis (Er.) Recorded outside Finland. Silfverberg 1977 NE 57:104.

Present number: $3564-14+56=3606$ spp.

\section{Lepidoptera}

Varis, V., Jalava, J. \& Kyrki, J. 1987 Check-list of Finnish Lepidoptera. - NE 67:49-118. 2320 spp.

Deletions

!Heliozela hammoniella Sorh. (sp.dub.). Kerppola \& al 1987 Baptria 12:63.

!Psyche betulina (Zell.) (f.d., = P. rotunda (Suom.)). Suomalainen 1990 Nota Lepidopterol. 13:229.

Coleophora tractella Zell. (f.d., = C. svenssoni Bald.). Baldizzone 1985 Alexanor 14:106.

Ectropisbistortata (Gze.) (=E.crepuscularia (D\&S.). Varis \& al. 1987 NE 67:84

Additions

!Trifurcula subnitidella (Dup.) Ka: Virolahti (Kaitala). Nieukerken 1990 Tijdschr. Entomol. 133:224.

Stigmella plagicolella (Staint.) N: Hanko. Laasonen \& Laasonen 1986 Baptria 11:45.

! - lappovimella (Svenss.) (sp.dist.) Ob, Ok, Ks, Lk, Le. Bruun 1988 NE 68:7. — not sp.dist.: Johansson \& Nielsen 1990 Fauna Entomol. Scand. 23:196.

!*Psyche $[$ Proutia] rotunda (Suom.) (betulina auct.). Finland. Suomalainen 1990 Nota Lepidopterol. 13:231.

Phyllonorycter pastorellus (Zell.) N. Varis \& al. 1987 NE 67:55; Kerppola, Kontuniemi \& Löfgren 1987 Baptria
12:65; Laasonen, Karvonen, Kyrki \& Peltonen 1988 Baptria 13:21; Kerppola \& Kontuniemi 1989 Baptria 15:20.

!Yponomeuta rorrellus (Hb.) N: Helsinki (Saarenoksa). Kullberg 1990 Baptria 15:115.

Prays ruficeps (Heinem.) (sp.dist.) Finland. Kyrki 1986 Baptria 11:28.

*Rhigognostis kuusamoensis Kyrki Ks: Kuusamo, Lk: Kittilä, Savukoski. Varis \& al. 1987:57 [as Rhigognostis sp.]; Kyrki 1989 Entomol. Scand. 19:451.

!Leucoptera lustratella (Herr.-Sch.) Kb: Kitee (Saarela). Kerppola \& Kontuniemi 1990 Baptria 15:54.

!Carcinaquercana (F.) Ab: Nousiainen (Teriaho). Kerppola \& Kontuniemi 1989:21.

Elachista cingillella Herr.-Sch. Ta: Valkeakoski (Saarela). Varis \& al. 1987:59; Kerppola \& Kontuniemi 1989:21.

Coleophora sibiricella Falk. Ab, Ta, Kb. Kerppola, Kontuniemi \& Löfgren 1986 Baptria 11:29; Kerppola, Kontuniemi \& Löfgren 1987:66; Kerppola \& Kontuniemi 1990:55.

* svenssoni Bald. (C.tractella auct. nec Zell.) Le, Li. Baldizzone 1985 Alexanor 14:106; Kerppola, Kontuniemi \& Löfgren 1986:66.

Hypatopa segnella (Zell.) (perfugella Jonass.) Ab: Rymättylä. Euranto 1986 NE 66:184.

!Metzneria ehikeella Gozm. Al: Jomala. Bruun \& Krogerus 1988 NE 68:27.

Anacampsis fuscella (Ev.) Ab, Ta. Varis \& al. 1987:63; Kerppola \& Kontuniemi 1989:23

Pristerognatha penthinana (Guen.) N: Borgå, Pellinge (Alvas). Varis \& al. 1987:66; Kerppola, Kontuniemi \& Löfgren 1987:68.

Prochoreutis solaris (Ersch.) Ab, N, Ka. Varis \& al. 1987:69; Kerppola, Kontuniemi \& Löfgren 1987:69; Kerppola \& Kontuniemi 1989:24; Kerppola \& Kontuniemi 1990:56.

Paraplatyptilia [Mariana] taprobanes (Feld. \& Rog.) Ka: Virolahti. Varis \& al. 1987:70; Kerppola, Kontuniemi \& Löfgren 1987:70.

!Pyrausta obsoletalis F. Al: Eckerö. Krogerus 1990 NE 69:215.

Catoptria permiaca (Peters.) N, Ka. Laasonen \& Laasonen 1986 NE 66:167; Kerppola, Kontuniemi \& Löfgren 1987:70; Kerppola \& Kontuniemi 1989:24.

*Eudonia aequalis Palm Sb, Ok, Lk. Palm 1986 Danmarks Dyreliv 3:184.

Perinephela lancealis (D\&S) N: Ekenäs, Jussarö (Nupponen). Varis \& al. 1987:74; Nupponen \& Nupponen 1987 Baptria 12:76.

!Iphiclides podalirius (Scop.) Ab: Turku (Mantere). Avanto 1988 NE 68:154

!Melanargia galathea (L.) Sa: Joutseno (Haapala). Marttila 1990 Baptria 15:73.

!Epirrhoe rivata (Hb.) N: Tvärminne. Kaila 1990 Baptria 15:1.

Eupithecia irriguata (Hb.) Ab: Korpo. Roslin $1987 \mathrm{NE}$ 67:215; Kaila \& Roslin 1987 Baptria 12:77.

! - ochridata Pink. Al, Ab, N, Ka. Kaila 1989 NE 69:39. 
! - cauchiata (Dup.) N: Tvärminne. Kaila 1990:2.

Ptilophoraplumigera (D\&S) N: Sipoo (Lepistö). Repo 1986 Baptria 11:82. Lepistö 1987 NE 67:215.

!Acontia lucida (Hufn.) N: Vantaa. Ojala 1988 NE 68:154.

Diachrysia tutti (Kostr.) (sp.dist.) Ab: Houtskär. Bruun 1987 NE 67:125-127.

Autographa buraetica (Staud.) N, Ka, Ta, Sa, Sb. Kerppola \& Mikkola 1987 NE 67:119-123; Repo 1987 Baptria 12:83; Peltonen 1988 Baptria 13:6; Somerma 1988 Baptria 13:42; Repo 1989 Baptria 14:28.

Atypha pulmonaris (Esp.) Ka: Virolahti (Kaitila, Holmberg \& Kontuniemi). Repo 1986 Baptria 11:84. Kontuniemi 1987 NE 67:215.

!Protexarnis squalida (Guen.) N: Borgå, Bodö (C.E.Lindfors). Lindfors, Mikkola \& Ahti 1989 NE 69:8.

!Xestia brunneopicta (Mats.) Ks: Kuusamo. Mikkola, Sinervirta \& Vaalamo 1989 NE 69:33.

Present number: $2306-4+36=2338$ spp.

\section{Diptera}

Deletions

Bezzia borealis Clastr. (= Probezzia seminigra (Panz.)). Remm 1988 Catal. Pal. Dipt. 3:13.

Dasyhelea aestiva $($ Winn. $)(=$ D. modesta $($ Winn.) ). ibid.:75

- longipalpis Kieff. (= D. modesta (Winn.)). ibid.:75.

Culicoides neglectus (Winn.) (spec.dub.). ibid.: 110.

Corynoneura longipennis Tobun. (= C. scutellata Winn.). Hirvenoja \& Hirvenoja 1988 Spixiana Suppl. 14:221.

Corynoptera geogenia Tuomik. $(=C$. boletiphaga (Lengersd.)). Gerbachevskaja-Pavluchenko 1986 Catal. Pal. Dipt. 4:24.

Dasineuranervorum (Kieff.) $(=$ D. salicis (Schr.)). Skuhrava 1986 Catal. Pal. Dipt. 4:162.

Oligotrophus alopecuri Reut. (= Dasineura alopecuri (Reut.)) (Listed twice in check list). Hackman 1980 NE 60:38,39.

Iteomyia major (Kieff.) (= I. capreae (Winn.)). Skuhrava 1986:174.

Megaselia eminens Schm.(=M.sordida (Zett.)). Disney 1985 Entomol. Mon. Mag. 121:246.

- semiscaura Schm. (= M. sordida (Zett.)). ibid.:246,

- subnitida (Lundb.) $(=$ M. lucifrons (Schm.)). Disney 1988 Entomol. Mon. Mag. 124:11.

- angustata (error for angusta) [Wood] (= M. pulicaria (Fall.)). Disney 1989 Handb. Ident. Brit. Ins. 10(8):44.

- angustifrons (Wood) $(=$ M. minor (Zett.)). Disney 1984 Entomol. Mon. Mag. 120:239.

- nudiventris (Wood) (= M. discreta (Wood)). Disney 1985:245.

- scutellariformis (Schm.) (= M. scutellaris (Wood)). ibid.:245.

Sepsis luteipes Mel. \& Spul. (f.d., = S. punctum (F.)). Pont 1990 Entomol. Fennica 1:154.

Limosina manicata Rich. (= Spelobia clunipes (Meig.)). Pitkin 1988 Handb. Ident. Brit. Ins. 10(5e):12.
Amobiasignata (Meig.) (f.d.,=A. oculata (Zett.). Pape 1987 NE 67:43.

Senotainia albifrons (Rond.) (f.d., = Actia crassicornis (Meig.)). ibid.:44.

Phronisellanasuta (Meig.) (f.d., =P. sannio (Zett.)). ibid.:44. Macronychia conica Rob.-Desv. Recorded outside Finland. ibid.: 44 .

Brachicoma borealis Ringd. (= B. devia (Fall.)). ibid.:44.

Blaesoxiphalaticornis (Meig.) (f.d.,=B.plumicornis (Zett.)). ibid.: 45 .

Helicophagella agnata (Rond.) (f.d.,=Exorista sp.). ibid.:45. Heteronychia dissimilis (Meig.) (f.d.). ibid.:45.

Parasarcophaga tuberosa (Pand.) (f.d., = P. portschinskyi Rohdend.). ibid.:46.

Sarcophagalehmanni Müll.(f.d.,=S. schulzi Müll.). ibid.:46.

Additions

Nephrotoma relicta Savtsh. Ks: Kuusamo (Bruun). Rautio 1986 NE 66:190.

- submaculosaEdw. Oa:Ilmajoki (Mukkala). Rautio 1987 NE 67:216.

Tipula alpinum (Bergr.) Ab: Turku (Mukkala). Rautio 1986 NE 66:189.

Cheilotrichia neglecta Lacksch. Ab: Salo. Rautio 1987 NE 67:216.

Dasyhelea modesta (Winn.) (aestiva (Winn.), longipalpis Kieff.). Remm 1988 Catal. Pal. Dipt. 3:75.

Serromyia subinermis Kieff. Ab. Borkent \& Bissett 1990 Syst. Entomol. 15:182.

Boreochlus thienemanni Edw. Li: Utsjoki (Huldén). Tuiskunen \& Lindeberg 1986 Ann. Zool. Fennici 23:364.

Arctopelopia melanosoma (Goetgh.) Le: Enontekiö, Li: Inari. ibid.: 365 .

Conchapelopia intermedia Fittk. Ks: Kuusamo. ibid.: 365.

Diamesa bertrami Edw. Le: Enontekiö. ibid.:365.

— serratosioi Will. Le: Enontekiö. ibid.:365.

- incallida (Walk.) Li: Utsjoki. ibid.:365.

— latitarsis (Goetgh.) (?) Le: Enontekiö. ibid.:365.

- spinacies Saeth. (?) Le: Enontekiö. ibid.:365.

— thienemanni Kieff. Li: Utsjoki. ibid.:365.

Potthastia pastoris (Edw.) Li: Inari. ibid.:365.

*Sympotthastia huldeni Tuiskunen Li: Utsjoki. Tuiskunen 1986 Ann. Entomol. Fennici 52: 78.

Acamptocladius reissi Cranst.\& Saeth. Li: Inari. Tuiskunen \& Lindeberg 1986: 366.

- submontanus (Edw.) Le: Enontekiö, Li: Inari. ibid.:366.

*Boreosmittia inariensis Tuisk. Ta: Kangasala, Li: Inari. ibid.:374.

* karelioborealis Tuisk. Ka: Vehkalahti, Kb: Ilomantsi, Li: Inari. ibid: 376

Bryophaenocladius dentatus (Karl) Li: Inari. ibid.:366.

- psilacrus Saeth. Le: Enontekiö, ibid.:366.

* saanae Tuisk. Le: Enontekiö. ibid.:376.

- tuberculatus (Edw.) Le: Enontekiö. ibid.:366.

Chaetocladius acuminatus Brund. Le: Enontekiö. ibid.:366.

*- crassisaetosus Tuisk. Le: Enontekiö, Li: Inari. ibid.:378.

— gracilis Brund. Le: Enontekiö. ibid.:366.

- maari Brund. Le: Enontekiö. ibid.:366.

— tenuistylis Brund. Le: Enontekiö, Li: Inari. ibid.366. 
Corynoneura magna Brund. Li: Inari. ibid.:366.

- gratias Schlee Ta: Hausjärvi. Hirvenoja \& Hirvenoja 1988 Spixiana Suppl. 14:218.

- arctica Kieff. "bis Lappland". ibid.:228.

*_ brundini Hirv. \& Hirv. "ganz Finnland". ibid.:228.

Cricotopus cumulatus Hirv. Le: Enontekiö. Tuiskunen \& Lindeberg 1986:367.

- pirifer Hirv. Li: Inari. ibid.:367.

— pulchripes Verr. Li: Inari. ibid.:367.

*Doncricotopus dentatus Tuisk. Sa: Valkeala, Le: Enontekiö, Li: Inari. Tuiskunen 1986 Ann. Entomol. Fennici 51:102.

Eukiefferiella boevrensis Brund. Le: Enontekiö. Tuiskunen \& Lindeberg 1986:367.

- brevicalcar (Kieff.) Le: Enontekiö, Li: Inari. ibid.:367.

- devonica (Edw.) Le: Enontekiö, Li: Inari. ibid.:367.

- sivertseni Aag. Li: Inari. ibid.:367.

Gymnometriocnemus brumalis Edw. Le: Enontekiö. ibid.:367.

Heleniella ornaticollis (Edw.) Li: Inari. ibid.:367.

Heterotanytarsus brundini Fittk. Le: Enontekiö, Li: Inari. ibid.:367.

Hydrobaenus spinnatis Saeth. Le: Enontekiö. ibid.:368.

Krenosmittia halvorseni (Cranst.\& Saeth.) Ob, Ks, Li. ibid.:384.

Limnophyes difficilis Brund. Li: Inari. ibid.:368.

Metriocnemus ursinus (Holmgr.) Le: Enontekiö. ibid.:368.

Nanocladius rectinervis (Kieff.) Le: Enontekiö, Li: Inari. ibid. 368 .

Orthocladius olivaceus (Kieff.) Le: Enontekiö, Li: Inari. ibid.:368.

- decoratus (Holmgr.) Li: Inari. ibid.:368.

- saxicola Kieff. Le: Enontekiö. ibid.:368.

Paracladius alpicola (Zett.) Le: Enontekiö. ibid.:368.

Paracricotopus uliginosus (Brund.) Le: Enontekiö, Li: Inari. ibid.:368.

*Parakiefferiella bilobata Tuisk. Ok: Vaala. Tuiskunen 1986 Ann. Zool. Fennici 23:179.

* fennica Tuisk. Sa: Punkasalmi, Le: Enontekiö, Li: Inari. ibid.: 181 .

*_ minuta Tuisk. Ka: Vehkalahti. ibid.:185.

- scandica Brund. Ta: Kangasala, Le: Enontekiö, Li: Inari. ibid.: 188 .

Parametriocnemus boreoalpinus Gow. Li: Utsjoki. Tuiskunen \& Lindeberg 1986:369.

Paraphaenocladius irritus (Walk.) Le: Enontekiö, Li: Inari. ibid.:369.

Psectrocladius bisetus Wulk. Li: Inari. ibid.:369.

- edwardsi Brund. (sp.dist.) Oa: Seinäjoki. Koskenniemi \& Paasivirta 1987 Entomol. Scand. Suppl. 29:242.

- platypus (Edw.) Oa: Seinäjoki. ibid.:242.

Pseudorthocladius curtistylus (Goetgh.) Li: Inari. Tuiskunen \& Lindeberg 1986:369.

— pilosipennis Brund. Li: Inari. ibid.:369.

Pseudosmittia recta (Edw.) Le: Enontekiö. ibid.:369.

- ruttneri Strenzke Le: Enontekiö, Li: Inari. ibid.:369.

*Psilometriocnemus europaeus Tuisk. Ka, Ta, Sa, Le. Tuiskunen 1986 Ann. Entomol. Fennici 51:101.

Rheocricotopus tirolus Lehm. Le: Enontekiö. Tuiskunen \& Lindeberg 1986:369.

Rheosmittia spinicornis Brund. Li: Utsjoki. ibid.:369.

Smittia betuletorum Edw. Le: Enontekiö. ibid.:370.
*Thienemanniapaasivirtai Tuisk. Le: Enontekiö(Paasivirta). ibid.:386.

*Tokunagaia parexcellens Tuisk. Le: Enontekiö. ibid.:387.

- rectangularis (Goetgh.) Le: Enontekiö. ibid.:370.

Tokunagayusurika jacutica Zver. Tb: Laukaa, Vatia. Meriläinen 1987 Biol. Res. Rep. Univ. Jyväskylä 10:90.

Tvetenia bavarica (Goetgh.) Le: Enontekiö. Tuiskunen \& Lindeberg 1986:370.

- calvescens (Edw.) Le: Enontekiö, Li: Inari. ibid.:370.

- verralli (Edw.) Le: Enontekiö. ibid.:370.

*Zalutschia mallae Tuisk. Le: Enontekiö, Li: Inari. ibid.:388.

Pagastiella orophila (Edw.) Le: Enontekiö, Li: Inari. ibid::371.

Parachironomus subalpinus Goetgh. Le: Enontekiö. ibid.:371.

Polypedilum albicorne (Meig.) Le: Enontekiö. ibid.:371.

- arundineti (Goetgh.) Li: Inari. ibid.:371.

Tanytarsus latiforceps (Edw.) Ab: Dragsfjärd, Vänö. ibid::372.

Exechia papyracea Stack. Kb, Ok, Ks. Ståhls 1990 NE 69:214.

Allodia pyxidiiformis Zaitz. N: Esbo, Le: Kilpisjärvi. Ståhls 1986 NE 66:190.

Mycetophila strigatoides Landr. Ab, N,Ok. Ståhls 1990:214.

Plastosciara brevicornis Tuomik. (accidentally omitted from 1980 check list) Tuomikoski 1957 Ann. Entomol. Fennici 23:14.

Bradysia tristicula (Winn.) (difformis Frey) (accidentally omitted from 1980 check list) Frey 1948 NE 27:61.

Scaptosciara myrmecophila Frey (accidentally omitted from 1980 check list). ibid.:87.

Harmandia cavernosa (Rubs.) (sp.dist.) Skuhrava 1986 Catal. Pal. Dipt. 4:260.

Platypalpus stackelbergi Koval. Ta: Forssa. Chvála 1989 Acta Univ. Carol., Biol. 32:286.

Pipizella brevis Luc. Ta: Jokioinen, Somero (H.Hippa). Kuznetzov 1987 Entomol. Obozr. 66:428.

Melangyna lucifera Niels. Ta: Urjala (Brander). Hedström 1990 Entomol. Tidskr. 111:137.

Sphaerophoria chongjini Bankowska Li: Ivalo (J.A.W.Lucas). Decleer 1989 Bull. Ann. Soc. R. Belge Entomol. 125:153.

*Dorylomorpha onegensis Albr. Ab: Karislojo (Frey \& Hellén). Albrecht 1990 Acta Zool. Fennica 188:122.

*_ lautereri Albr. Oa: Petalax (Lindberg). ibid.: 124.

Cephalops germanicus (Acz.) Ab: Salo, Le: Kilpisjärvi. De Meyer \& Backeljau 1990 NE 69:200.

- subultimus Coll. Sa: Joutseno, Savonlinna, Tb: Keuruu. ibid.:201.

— semifumosus (Kow.) Ab: Lojo, Sa: Joutseno. ibid.:201.

Sepsis biflexuosa Strobl $A b$ : Korpo, Nagu, Ks: Kuusamo. Pont 1990 Entomol. Fennica 1:154.

- thoracica (Rob.-Desv.) Ab: Korpo Jurmo. ibid.:154.

Ortalischema albitarse (Zett.) Sa: Punkaharju. ibid.:154.

Themira biloba Anderss. $N$ : Helsinki. ibid.:154.

- germanica Duda $A b, N, S t, T a$. ibid.: 154 .

- lucida (Staeg.) Al, Ab, N, Ta, Sa, Oa, Tb, Ob, Ks, Li. ibid.: 154 .

- malformans Mel. \& Spul. Lk: Muonio, Li: Utsjoki. ibid.: 154 .

- paludosa Elb. Ab, N, St, Sb, Ks. ibid.:154. 
Campichoeta obscuripennis (Meig.) N. Chandler 1987 Entomol. Scand, 18:46.

Diastata ornata Meig. N, Ta, Lk, Li. ibid.:20.

* - boreonigra Chandler Ok, Ks, Lk. ibid.:22.

- costata Meig. $N, T b$. ibid.:28.

*_ flavicosta Chandler N, Sb, Ks, Lk. ibid.:29.

Agromyza demeijerei Hend. N: Helsinki. Vikberg 1986 NE 66:190.

*Strobilomyia sibirica Michels. Ab, N, Ka, St, Ta, Sa, Kb, Om, Ok. Michelsen 1988 Syst. Entomol. 13:297; Pulkkinen 1989 Ann. Entomol. Fennici 55:42.

- infrequens (Ackl.) Ab, N, Sa, Kb, Ok. Michelsen 1988:301; Pulkkinen 1989:42.

- laricicola (Karl) Ab, N, Sa. Michelsen 1988:304; Pulkkinen 1989:42.

Azelia monodactyla Loew Sa: Punkaharju. Pont 1990 NE 69:215.

- trigonica Henn. Sa: Punkaharju. ibid.:215.

*Phaonia taigensis Zinovj. N: Helsinki (B.Mamaev). Zinovjev 1987 Entomol. Obozr. 66:440.

Myospila bimaculata (Macq.) Sa: Punkaharju. Pont 1990 Entomol. Fennica 1:154.

Amobia oculata (Zett.) (signata auct. nec (Meig.)) St: Yläne (Sahlberg). Pape 1987 NE 67:43.

Miltogramma germari Meig. Sa: Joutseno (Thuneberg). ibid.:43.

- villeneuvei Verves Sa: Joutseno, Lauritsala(Thuneberg). ibid.:43.

Oebalia minuta (Fall.) Ta: Vanaja (Valkeila), ibid.:44

Phronisella sannio (Zett.) (nasuta auct. nec (Meig.)) Finland. ibid.:44

Metopia grandii Vent. Ab, Sa, Om. ibid.:44.

Heteronychia proxima (Rond.) N: Espoo (Tuomikoski). ibid.:45.

- vicina (Macq.) Al: Åland (Palmén). ibid.:45.

Thyrsocnema kentejana Rohdend. (lapponica Tiens.) (sp.dist.). ibid.:45.

Present number: $5067-28+131=5170 \mathrm{spp}$

\section{Hymenoptera}

Vikberg, V. 1986. A checklist of aculeate Hymenoptera of Finland (Hymenoptera, Apocrita Aculeata). - NE 66:65-85. - $601 \mathrm{spp}$.

Deletions

Trichiosoma boreale Guss. (= T. vitellinae $(\mathrm{L})$.$) Viitasaari$ 1989 Ann. Entomol. Fennici 55:112.

Pamphilius depressus (Schr.) (=P. vafer (L.)) Achterberg \& Aartsen 1986 Zool. Verhand. 234:44.

Sterictophora sorbi Kontun. (= S. geminata (Gmel.)) Koch 1988 Entomol. Abh. (Dresd.) 52:42.

Monophadnoides puncticeps (Konow) (= Claremontia confusa (Konow)) Koch 1988 Deutsche Entomol. Zeitschr. N.F. 35:315

Pontania crassipes (Thoms.) (=P. viminalis (L.)) Kopelke 1989 Senckenbergiana biol. 69:35.

Adelognathus fasciatus Thoms. (= A. tetracinctorius (Thunb.)) Kasparyan 1986 Tr. Zool. Inst. 159:51
Plectiscidea melanocera (Först.) (f.d., =P. moerens (Först.) \& P.amicalis (Först.)) van Rossem 1987 Tijdschr. Entomol. 130:68, 71.

Diospilus oleraceus Halid. (=D. capito (Nees)) Tobias 1986 Opred. faune SSSR 145:157.

Charmon cruentatus Halid. (= C. extensor (L.)) ibid:274.

Agathis arcuata (Reinh.) (= Microdus conspicuus Wesm.) Nixon 1986 Bull. Brit. Mus. Nat. Hist. (Entomol.) $52: 255$.

Earinus delusor (Wesm.) (= E. gloriatorius (Panz.)). ibid.:228.

- nitidulus (Nees) (=E. elator $(\mathrm{F})$.$) . ibid.:227.$

- thoracicus (Nees) $(=$ E. elator $(\mathrm{F})$.$) . ibid.:227.$

Apanteles difficilis (Nees) (=A. cajae (Bouché)) Papp 1987 Ann. Hist.-Nat. Mus. Natn. Hung. 79:208.

- nigrinervis (Thoms.) (=A.jucundus Marsh.). ibid.:235

Rhysipolis caudatus (Thoms.) (= R. decorator (Halid.)) Tobias 1986:67.

- obscuripes (Thoms.) (=R. meditator (Halid.)). ibid.:68

Ontsira ignea (Ratz.) (= O. imperator (Halid.)). ibid.:43.

Ecphylus hylesini (Ratz.) (=E. silesiacus (Ratz.)). ibid.:31.

- pinicola Hedqv. (= E.silesiacus (Ratz.)). ibid.:31.

Acrisis koponeni Tob. (= A. brevicornis Hellén) Belokobylski 1990 Vestn. Zool. 1990(6):36

Bracon pumilionis Rom. (= B. variator Nees). Tobias 1986:134.

Ipobracon borealis (Hellén) (=I. nigrator (Zett.)). ibid.:107.

Alloxysta crassa (Camer.) (= A. macrophadna (Hart.)) Fergusson 1986 Handb. Ident. Brit. Ins. 8(1c): 10.

- perplexa(Camer.) (=A.macrophadna(Hart.)). ibid.:10.

- piceomaculata (Camer.) (= A. macrophadna (Hart.)). ibid.: 10 .

- defecta (Hart.) (= A. fulviceps (Curt.)). ibid.:10.

- castanea (Hart.) (= A. fulviceps (Curt.)). ibid.:10.

- nigriventris (Thoms.) (= A. fulviceps (Curt.)). ibid.: 10 .

- circumscripta (Hart.) (= A. victrix (Westw.)). ibid.:11.

- curvicornis (Camer.) (= A. victrix (Westw.)). ibid.:11.

- halterata (Thoms.) (= A. pedestris (Curt.)). ibid.:11.

Melanips femoralis Camer. (= M. opacus (Hart.)). ibid.: 12.

Encyrtus obscurus Dalm. (=E. infidus (Rossi)) Trjapitzin 1989 Opred. faune SSSR 158:372.

Bethylus apteryx Kieff. (f.d.) Vikberg 1986 NE 66:61.

Chrysis westerlundi Lins. (= C. bicolor Lep.). ibid.:62.

- gribodoi $\mathrm{Ab}$. (f.d.). ibid.:62.

- indigotea Duf. \& Perr. Recorded outside Finland. ibid.:62.

Pterocheilus phaleratus (Panz.) Recorded outside Finland. Pulkkinen 1926 NE 6:25; Vikberg 1986 NE 66:73.

!Symmorphus mutinensis (Bald.) $(=$ S. bifasciatus (L.)) Cumming 1989 Mem. Entomol. Soc. Canada 148:57.

Ancistrocerus gazella (Panz.) (f.d., = A. ichneumonideus (Ratz.)) Vikberg 1986:74.

!Spilomena differens Blüthg. (=S.curruca(Dahlb.)) Dollfuss 1986 Ann. Naturh. Mus. Wien 88/89(B):488.

!Nomada montana (Mocs.) (tormentillae Alfk.) $(=N$. roberjeotiana Panz.) Schwarz 1986Entomofauna 7:460.

Additions

Sirex atricornis Kjellander (sp.dist.) Ok: Suomussalmi, "Lapland". Viitasaari \& Midtgaard 1989 Ann. Entomol. Fennici 55:108. 
Trichiosoma laterale Leach Ta: Lammi (Kontuniemi). Viitasaari 1989 Ann. Entomol. Fennici 55:113.

- crassum Kby. (jakovleffi Konow) "Northern Fennoscandia". ibid.:116.

Pamphilius festivus Pes. \& Pes. Ta: Lammi (Kontuniemi). Shinohara \& Taeger 1990 Bull. Nat. Sci. Mus. (Zool.), Tokyo 16:90.

Pontania herbaceae (Camer.) (aquilonia Bens.) (sp.dist.) Kopelke 1989 Senckenbergiana biol. 69:44.

- polaris Mal. (sp.dist.). ibid.:61.

Endromopoda nigricoxis (Ulbr.) (sp.dist.) Fitton, Shaw \& Gauld 1988 Handb. Ident. Brit. Ins. 7(1):47.

Tromatobia forsiusi (Hellén) (sp.dist.). ibid.:57.

Delomerista novita europa Gupta Ab, Ka,Ta,Sa Jussila 1989 NE 69:75

- pfankuchi (Brauns) Li: Utsjoki. ibid.:75.

- frigida Kasp. Li: Utsjoki (Koponen \& Linnaluoto). ibid.:76.

Dyspetes arrogator Heinr. Ka: Virolahti. ibid.:76.

Aclastus borealis (Boh.) Le: Enontekiö. ibid.:78.

- transversalis Horstm. Kl: Parikkala. ibid.:78.

- Iongicauda Horstm. Ab: Turku, Sauvo, Oa: Vaasa. ibid.:78.

Dichrogaster nigrithorax Horstm. $N$ : Helsinki (Townes), St: Yläne (Jussila). Townes 1983 Mem. Amer. Entomol. Soc. 35:113; Jussila 1989:78.

— perlae (Doumerc) Al:Vårdö(Koponen). Jussila 1989:79.

- heteropus (Thoms.) Al, Ab, N. ibid.:79.

Gelis albipalpus (Thoms.) N: Nurmijärvi, Ta: Heinola. Koponen 1988 NE 68:154.

- fasciitinctus (D.T.) Sa: Mikkeli. ibid.:154.

- elymi (Thoms.) Al, Ab, Ka, Oa, Tb. Jussila 1989:79.

- linearis (Först.) St: Yläne. ibid.:79.

Isadelphus coriarius (Taschb.) Ab, Ka, Ta, Sa. Jussila 1986 NE 66:127.

Charitopes gastricus (Holmgr.) N: Helsinki. Townes 1983:67.

Endasys kriegeri (Habermehl) (sp.dist.) Ab,N. Jussila 1986 NE 66:125.

*Amphibulus fennicus Sawon. N: Helsinki (Ranin, Perkiömäki \& Koponen). Sawoniewicz 1990 Ann. Zool. (Warsz.) 43:288.

Bathythrix argentatus (Grav.) Ab: Nauvo, Sauvo. Jussila 1986 NE 66:125.

- maculatus(Hellén)(sp. dist.). Horstmann 1990 Entomol. Fennica 1:183.

Theroscopus faciator (Aub.) Ta: Urjala (Brander). Jussila 1986 NE 66:127.

Phygadeuon rugipectus Thoms. (sp. dist.). Jussila 1989:78.

Stilpnusoligomerus (Först.) N: Helsinki (Koponen), Porvoo (Koponen), Sa: Ristiina (Koponen). Jussila 1987 Ann. Entomol. Fennici 53:6.

- dryadum Curt. Al: Lemland, Ta: Lempäälä (Sotavalta). ibid.:7.

- montanus (Först.) Ta: Ruovesi (Saaristo). ibid.:8.

* bottnicus Jussila Al, Ab, N, Sa,Oa, Om. ibid.:9.

- rectangulus Roman Al, Ab, N, Ta. ibid.: 14.
- parvulus Först. $A l, A b, N$, Ta. ibid.: 14.

Apsilops tenebrosus Hellén (sp. dist.). Horstmann 1990 Entomol. Fennica 1:183.

Caenocryptus melanarius (Hellén) (sp. dist.). ibid.:183.

Lissonota extrema Hedw. Ab, N. Jussila 1989 NE 69:77

Synoecetes anterior (Thoms.) Ta, Tb, Ok, Li. Jussila 1986 NE 66:127.

Synodites amoenus (Roman) Li: Utsjoki (Hanhimäki). Jussila 1989:77.

Dusona humilis (Först.) Al: Lemland. Jussila 1986 NE 66:127.

— thomsoni Hinz Ab: Rymättylä (Euranto), Ka: Virolahti. ibid.: 127.

- angustifions (Först.) Ab: Sauvo. ibid.:128.

- stenocara (Thoms.) Ka: Virolahti. ibid.:128.

- adriaansei (Teun.) Ab: Sauvo, Ta: Lempäälä (Sotavalta). ibid.: 128.

- disclusa (Först.) Ok: Kuhmo. ibid.:128.

Tranosemella citrofiontalis (Hedw.) Ka, Oa, Tb. Jussila 1986 NE 66:128.

Enytus appositor (Aub.) Al, Ka. Jussila 1986 NE 66:128.

*Eriborus obscuripes Horstm. Ab: Houtskär (Bruun). Horstmann 1987 Nachr. Bl. Bayer. Entomol. 36:64.

Plectiscidea moerens (Först.) (sp.dist.) van Rossem 1987 Tijdschr. Entomol. 130:68.

— amicalis (Först.) (sodalis (Först.)) (sp.dist.). ibid.:71.

Dyscoletes lancifer (Halid.) Li: Utsjoki (S.Koponen). Achterberg 1988 Zool. Verhand. 249:32.

Orgilus temporalis Tob. $A b$ : Nystad, $N$ : Ekenäs, Lovisa. Taeger 1989 Die Orgilus-Arten der Paläarktis, Berlin, p. 197.

Earinus elator (F.) (nitidulus (Nees), thoracicus (Nees)) Nixon 1986 Bull. Brit. Mus. Nat. Hist. (Entomol.) 52:227.

Apanteles villanus Reinh. Finland. Papp 1986 Ann. Hist.Nat. Mus. Natn. Hung. 78:228.

- ofella Nixon Finland. ibid.:231.

- berberis Nixon Finland. ibid.:231.

- orestes Nixon Finland. ibid.:232.

* peltoneni Papp N: Helsinki, ex Acronicta leporina (E.Peltonen). Papp 1987 Ann. Hist.-Nat. Mus. Natn. Hung. 79:241.

*Chelonus jacobsoni Tobias Sa: Rajala (Adelung). Tobias 1986 Opred. faune SSSR 145:315.

Alysia brachycera Thoms. $N$ : Helsinki (Townes \& Townes). Wharton 1986 Syst. Entomol. 11:468.

Phaenocarpa canaliculata Stelf. Finland. Tobias 1987 Opred. faune SSSR 147:141.

* - chiastochetae Fisch. Ks: Oulanka (Pellmyr). Fischer 1990 Ann. Naturh. Mus. Wien 91 B:114.

- ruficeps (Nees) Lk: Pallastunturi (Mihaly). ibid.:120.

* pellmyri Fisch. Ks: Oulanka (Pellmyr). ibid.: 123.

Pambolus pallipes (Först.) Finland. Belokobylskij 1986 Tr. Zool. Inst. 159:34.

Dendrosoter flaviventris Först. (sp.dist.) Tobias 1986:41.

Rhyssalus longicaudis (Tob.\& Belok.) Finland. Tobias 1986:37. 
Bracon fumipennis Thoms. (sp.dist.). ibid.:131.

Ephedrus nacheri Quilis N: Helsingfors (Hellén). Gärdenfors 1986 Entomol. Scand. Suppl. 27:74.

*Pseudopauesia prunicola Halme N, Sa, Oa. Halme 1986 Ann. Entomol. Fenn 52:21.

*Gasteruptionmargotae Madl St: Suoniemi(Saarinen). Madl 1987 Entomofauna 8:225.

Melanips austriaca (Tavares) Finland. Pulkkinen 1989 Ann. Entomol. Fennici 55:44.

Endomychobius endomychi (Walk.)Ta: Pälkäne (J.Kangas). Vikberg 1986 NE 66:189.

Pannoniella sexramosa Erdös Ta: Sysmä. Vikberg 1986 NE 66:189.

Arthrolytus ocellus Walk. Ta: Janakkala. Vikberg 1986 NE 66:189.

Eumacepolus pulcher Grah. Ta: Janakkala. Vikberg 1986 NE 66:189.

Eucoccidophagus kareliamus Shark. Finland. Trjapitzin 1989 Opred. faune SSSR 158:113.

Charitopus obscurus (Erd.) Finland. ibid.:114.

Coccidoxenoides peregrinus (Timberl.) Finland. ibid.:117. Anagyrus securicornis Domen. Finland. ibid.: 131.

- schoenherri (Westw.) Finland. ibid.:134.

Microterys sylvius (Dalm.) Finland. ibid.:164.

- [Aschitus] problematicus (Hoffer) N: Nurmijärvi. Jensen 1989 Entomol. Scand. 19:302.

_ duplicatus (Nees) Finland. Trjapitzin 1989:179.

- [Aschitus] aeneiventris (Walk.) Ta: Janakkala (Vikberg), $K b$ : Kontiolahti (Vikberg). Jensen 1989:299.

Trichomasthus [Aschitus] zarina (Walk.) N, Ta. ibid.:315.

— danzigae Trjap.Ta: Janakkala(Vikberg), $K b$ : Polvijärvi (Vikberg), Kontiolahti (Vikberg). Jensen \& Sharkov 1989 Entomol. Scand. 20:46. Trjapitzin 1989:189.

Metablastothrix truncatipennis (Ferr.) Finland. Trjapitzin 1989:201.

Ooencyrtus solidus Khlop. Finland. ibid.:207.

Helegonatopus nikolskajae Hoff. Finland. ibid.:214.

*Aenigmaphycus paluster Shark. \& Voin. Kb: Juuka (Koponen), Le: Enontekiö (Koponen). Sharkov \& Voinovich 1988 Entomol. Obozr. 67:827.

Metaphycus zebratus (Merc.) Finland. Trjapitzin 1989:238.

- stagnarum Hoff. Finland, ibid.:239.

- nitens (Kurdj.) Finland, ibid.:241.

- ater (Merc.) Finland. ibid.:242.

Mesaphycus picearum (Erd.) Finland. ibid.:247.

* Psyllaephagus hyperboreus Trjap. Ab: Vihti (Koponen). Trjapitzin 1986 Tr. Zool. Inst. 159:57.

- vendicus (Erd.) Finland. Trjapitzin 1989:262.

Cercobelus jugaeus (Walk.) Finland. ibid.:266.

*Mayridia helleni Trjap. N: Helsinki, Ta: Hämeenlinna, Lammi. ibid.:283.

Ectroma arenarium (Erd.) Finland. ibid.:286.

Cheiloneurus rediculus (Trjap. \& Khlop.) Finland.ibid.:310.

*Bothriothorax koponeni Khlopunov Sa: Ruokolahti (Koponen), Ob: Pudasjärvi(Koponen). Khlopunov 1984 Zool. Zh. 63:940.
Trjapitzinellus obscurus (Merc.) Finland. Trjapitzin 1989:327.

- nigricornis Hoff. Finland. ibid.:328.

Agekianellanotatifemur (Hoff.) (koponeni Trjap.) N: Vantaa (Koponen). Trjapitzin 1981 Zool.Zh. 60:787. Trjapitzin 1989:328.

Cerchysiellacentennalis (Erd.) Finland. Trjapitzin 1989:329.

Copidosoma clavigerum (Merc.) Finland. ibid.:345.

- $\operatorname{aretas}$ (Walk.) Finland. ibid.:348.

- agrotis (Fonsc.) Finland. ibid.:351.

Coelopencyrtus callidii (Janss.) Finland. ibid.:360.

Homalotyloidea novickyi Hoff. Finland. ibid.:366.

Isodromus flaviceps (Dalm.) Finland. ibid.:369.

Encarsia aleurochitonis (Merc.) N, Ka, Ta. Huldén 1986 NE 66:18.

*_ borealis Huldén Ab, N, Ta, Sa, Kl, Kb. ibid.:18.

- margaritiventris (Merc.) N: Helsinki, Elimäki.ibid.:18.

*_ moffsi Huldén N, Ta, Sa. ibid.: 19.

*Eretmocerus zippanguiphagus Huldén N,Ta. Huldén 1986 NE 66:19.

*Euderomphale secreta Huldén N, Ta. Huldén 1986 NE 66:20.

Derostenus punctiscuta Thoms. Ab, N, Sa,Tb. Hansson 1986 Entomol. Scand. 17:317.

Chrysonotomyia germanica (Erdös) Finland. Hansson 1990 Entomol. Scand. 21:35.

- lyonetiae (Ferr.) Finland. ibid.:36.

Neochrysocharis aratus (Walk.). Finland. ibid.:42.

- arvensis Grah. Finland. ibid.:43.

- formosa (Westw.) Finland. ibid.:47.

- nunbergi (Szczep.) Finland. ibid.:49.

Chrysocharis chlora Grah. Finland. ibid.:50.

*Amitus croesus Huldén N: Esbo. Huldén 1986 NE 66:22.

- minervae Silv. N: Helsinki. ibid.:23.

Arachnospila westerlundi (Moraw.) (bohemabnormis (Wolf)) Sa: Rantasalmi (Westerlund). Valkeila 1986 NE 66:62.

!Symmorphus allobrogus (Sauss.) (bifasciatus auct.) Al-Ks. Cumming 1989 Mem. Entomol. Soc. Canada 148:83.

!Ancistrocerus gazella (Panz.) Al: Eckerö. Pekkarinen \& Perkiömäki 1987 NE 67:207.

Spilomena beata Bluthg. Finland. Vikberg 1986:76.

Psenulus gussakovskiji (v. Lith) (sp.dist.) Finland. ibid.:75.

Trypoxylon minus Beaum. (sp.dist.) Finland. ibid.:76.

- medium Beaum. (sp.dist.) Finland. ibid.:76.

!Hylaeus pfankuchi (Alfk.) Al: Hammarland. Lomholdt 1989 NE 69:57.

!*Nomada fusca Schwarz Ab, N, Ta. Schwarz 1986 Entomofauna 7:434.

Accidentally omitted from check-list

!Crossocerus walkeri (Shuck.) Forsius 1935 Enum. Ins. Fenniae 2(1): 10.

Present number: $5547-43+140=5644$ spp. 